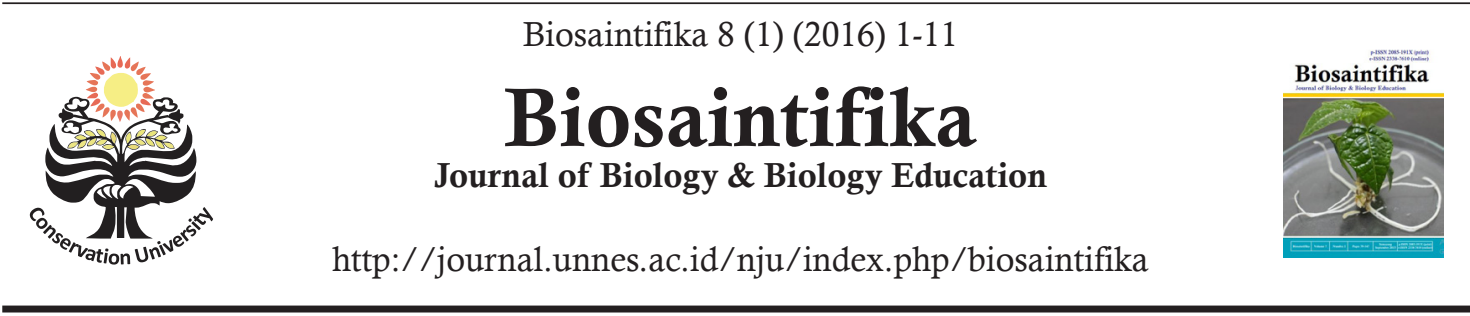

\title{
Knowledge Understanding and Utilization of Medicinal Plants by Local Community Tompu District of Kaili, Sigi Biromaru, Central Sulawesi
}

\section{Slamet Ifandi, Jumari, Sri Widodo Agung Suedy}

DOI: 10.15294/biosaintifika.v8i1.4529

Faculty of Science and Mathematics, Diponegoro University, Indonesia

\section{History Article}

Received 25 November 2015 Approved 15 January 2016 Published 9 March 2016

\section{Keywords:}

Knowledge; Medicinal Plants; Tompu community

\begin{abstract}
Kaili is one of the ethnic region in Central Sulawesi which saves a lot of cultural values and traditions. As a traditional community, their life is very dependent upon natural resources contained in the environment. They still have knowledge, traditional culture, treatment and utilization system against various types of plants. The purpose of the study was to examine the knowledge understanding and utilization of medicinal plants by local community Tompu District of Kaili. Data knowledge and utilization were collected through interview, literature study, exploratory survey methods, PEA (participatory ethnobotanical appraisal), questionnaire and from interviews with the informants. The results from interviews showed that of public knowledge is still based on the traditional concept. Based on the results identifications obtained by (90 species). As many as six species medicinal plants to often used the Tompu community are Euphorbia hirta L. Phyllanthus niruri L. Ageratum L. Blumea conyzoides balsaminifera L. (DC). Kleinhovia hospita $L$ and Tabernaemontana pandacaqui. The benefits of this research to the development of science is expected to be complete scientific data regarding the utilization of medicinal plants natural resources on Tribal society Kaili in Tompu.
\end{abstract}

\section{How to Cite}

Ifandi, S., Jumari, J., \& Suedy, S. W. A. (2016). Knowledge Understanding and Utilization of Medicinal Plants by Local Community Tompu District of Kaili, Sigi Biromaru, Central Sulawesi. Biosaintifika: Journal of Biology \& Biology Education, $8(1), 1-11$.

(C) 2016 Semarang State University

Correspondence Author:

p-ISSN 2085-191X

Prof. Soedarto, SH, Tembalang, Semarang 50275

e-ISSN 2338-7610 


\section{INTRODUCTION}

Plants play an important role in human life as a supplier of plant material necessities such as food, clothing, and medicine. Every society in every region has been using a variety of useful plants in their environment. In Indonesia, medicinal use of natural materials has been done by the ancestors' and it has been passed down from one generation to the next. A study of the interrelationships and linkages between cultural communities with the use of biological resources has called ethnobotany (Nasab \& Khosravi, 2014).

There are some researchers on knowledge of medicinal plants in Indonesia. The researchers also conducted a study and found the different types of potential medicinal plants. However, the existence of trends and public awareness on using natural medicine (back to nature) is an obvious fact that now people have started to gradually abandons the use of modern medicine. Local communities in district of Riau Province Pangean tribe utilizing plant of Zingiberaceae in everyday life as a traditional medicine (Hartanto et al, 2014). According to the evidence and some information, it showed that since ancient times, human beings are capable of treating a variety of diseases by utilizing the plant. Humans tend to mimic the behavior of animals regarding the use of plants including treatment. Even up to modern times humans still use animals to test drugs that were newly found (Yuniati \& Alwi, 2010).

Local communities in Kaili tribe, Tompu, Central Sulawesi also have made use of plants as traditional medicine. This empirical knowledge and experience are basic information that should be explored and assessed to be developed and exploited, in particular to explore renewable ingredients. The use of medicinal plants and the perception of healthy and sick concepts are formed through a process of socialization that has been passed down from generation to generation which trusted and believed to be the source of truth (Rahayu et al., 2006). Tompu society is a traditional society with a lot of cultural values and unique traditions. Culture is seen from the form of a society that still relies on the utilization of natural resources. As the rural communities, they have a fairly good level of knowledge about the concept of healthy, sick, the cause of the disease and the utilization of the potential types of medicinal plants, and food. The knowledge of Tompu society about medicinal plants has been passed down from generation to generation and still maintained until now. Knowledge is seen as a form of culture and heritage that must be main- tained and preserved. Therefore, traditional knowledge is not extinct. On this basis, it is necessary to do research aimed at assessing the knowledge of Tompu society in the utilization of medicinal plants traditionally.

\section{METHODS}

This study was conducted in January-May 2015. The research site was located in Tompu Loru and Ngata Baru Village, Biromaru District, Sigi, Central Sulawesi, Indonesia. There were 60 respondent of the study. The selection of the respondent based on the characteristics of the age between 20-50 years and above. The selection of respondents based on their status in society is obtained upon the recommendation of the customs and expert treatment. Educational level of respondents, in General, is still very low.

The research is divided into two stages. The first stage of the inventory level of public knowledge about medicinal plants using the method of the exploratory survey through in deep and open ended interviews from the informant (Jumari et al, 2012). Data supported a assessment approach with Participatory Ethnobotany Appraisal Method (PEA) that research get involved in the daily activities of the Community in Tompu (Martin, 2004). The second stage of the activities utilization of medicinal plants which include head to the composition of the material using a semi-structured interview. Data of the all of the use medicinal plants is presented in tabulated and analyzed the qualitative descriptive (Shanthi et al., 2014).

\section{RESULT AND DISCUSSION}

\section{General state of research sites}

Tompu is administratively located in two villages, i.e. Desa Loru and Ngata Baru with coverage area $\pm 6220.34 \mathrm{ha}$. Geographic position lies between $0^{\circ} 50^{\prime}-0^{\circ} 55^{\prime}$ south latitude and $119^{\circ} 58^{\prime}$ $120^{\circ} 00^{\prime}$ east longitude, the altitude is between $0-1750$ meters above sea level. Tompu is within $\pm 12 \mathrm{~km}$ from the capital city of the Province and $\pm 15 \mathrm{~km}$ distance from the Capital District. The total population of Kaili tribe residing in Tompu in 2015 is around 359 people, which includes about 42 head of the family is Loru village and 52 families in Ngata Baru. Tompu people occupation and livelihood is farming, hunting and utilizing the forest products for particular purpose, for example using it as medical supplies. A health facility in this village is a community health clinic that is handled by midwives and nurses. Health 
facility is intended to provide facilities for the local community in obtaining health care without reducing the role of local traditional medicine.

\section{Level of Knowledge of Tompu Society about Medicinal Plants}

Traditional knowledge and the use of plants by people in Tompu require in-depth assessment process. In fact, it has been proved that traditional knowledge contributes to the progress of science and technology (Jumari, 2012). According to Josi et al. (2004), it was found that local knowledge as a result of learning, thinking, and perception as well as the basis for estimating future events.

In general, the process of knowledge transfer of medicinal plants by the community in Tompu was done orally, secretly and closely. Not all people of Tompu in research sites have the same level of knowledge in the use of medicinal plants. According to Sando, the more it is influenced by education level, personal knowledge and the intensity of the use of medicinal plants, then more the tendency of public knowledge about a many medicinal plants are also. Information knowledge of medicinal plants in the society can only be delivered and given to certain people, such as children and their families. Knowledge of society is the cultural heritage and traditions that must be maintained and kept confidential. Hariyadi, (2011) stated that the use of plants for traditional medicine in public still rely on legacy knowledge passed down through generations. The knowledge can be gradually eroded due to acceptance of each person that will be different in the transfer process of knowledge on the utilization of medicinal plants. Tompu society has a diverse knowledge level in the use of medicinal plants. It is influenced by a person's level of knowledge. In the process of transformation, Tompu society knowledge of medicinal plants is only revealed to those who are older as traditional leaders, sando or shaman, and members of his family who are believed to pass on this knowledge. The results showed that the 90 species of plants obtained were utilized by Tompu community (Table 1).

Table 1. Medicinal plant utilization category of Kaili Tribe in Tompu Village

\begin{tabular}{|c|c|c|c|c|c|c|}
\hline Category of Disease & & Composition & & Organ & $\begin{array}{c}\text { Concoction } \\
\text { Technique }\end{array}$ & Usage \\
\hline Type of Treatment & Local Name & Scientific Name & Family & & & \\
\hline $\begin{array}{l}\text { Treatment of diar- } \\
\text { rhea and constipa- } \\
\text { tion }\end{array}$ & Kaleta even & Acalypha indicia L. & Euphorbiaceae & Root & $\begin{array}{l}\text { Boiling tech- } \\
\text { nique }\end{array}$ & Drink \\
\hline $\begin{array}{l}\text { Curing heartburn, } \\
\text { anti-poison of insect } \\
\text { bites, and traditional } \\
\text { rituals materials. }\end{array}$ & Salembangu & $\begin{array}{l}\text { Ageratum conyzoi- } \\
\text { des L. }\end{array}$ & Asteraceae & Leaf & $\begin{array}{l}\text { Boiling tech- } \\
\text { nique }\end{array}$ & Drink \\
\hline $\begin{array}{l}\text { Treatment of dys- } \\
\text { entery }\end{array}$ & Bealu & $\begin{array}{l}\text { Aleurites moluccana } \\
\text { Willd. }\end{array}$ & Euphorbiaceae & Bark & $\begin{array}{l}\text { Mashing the } \\
\text { bark, squeez- } \\
\text { ing the water }\end{array}$ & Drink \\
\hline $\begin{array}{l}\text { Treatment of } \\
\text { stomachache and } \\
\text { digestive function }\end{array}$ & Sanggulera & Averoa bilimbi $\mathrm{L}$. & Oxalidaceae & $\begin{array}{l}\text { Leaf } \\
\text { and } \\
\text { fruit }\end{array}$ & $\begin{array}{l}\text { Boiling tech- } \\
\text { nique }\end{array}$ & Drink \\
\hline $\begin{array}{l}\text { Treating diarrhea } \\
\text { and other chronic } \\
\text { diseases }\end{array}$ & Sivumboa & $\begin{array}{l}\text { Blumea balsa- } \\
\text { minifera (L.)DC. }\end{array}$ & Asteraceae & Leaf & $\begin{array}{l}\text { Boiling tech- } \\
\text { nique }\end{array}$ & Drink \\
\hline $\begin{array}{l}\text { Helps the process of } \\
\text { digestion and treat- } \\
\text { ment of injuries }\end{array}$ & Toboyo & $\begin{array}{l}\text { Cucurbita muscata } \\
\text { L. }\end{array}$ & Cucurbitaceae & Flower & $\begin{array}{l}\text { Boiling } \\
\text { technique and } \\
\text { then added by } \\
\text { coconut water }\end{array}$ & Drink \\
\hline Curing dysentery & Gampiasu & Desmodium sp. & Fabaceae & Leaf & $\begin{array}{l}\text { Boiling tech- } \\
\text { nique }\end{array}$ & Drink \\
\hline $\begin{array}{l}\text { Curing dysentery, } \\
\text { anti-poison, fever, } \\
\text { and asthma. }\end{array}$ & Mantalalu & Euphorbia hirta L. & Euphorbiaceae & $\begin{array}{l}\text { All } \\
\text { parts }\end{array}$ & $\begin{array}{l}\text { Boiling } \\
\text { technique and } \\
\text { then added } \\
\text { by honey and } \\
\text { milk }\end{array}$ & Drink \\
\hline
\end{tabular}


Slamet Ifandi, et al. / Biosaintifika 8 (1) (2016) 1-11

\begin{tabular}{|c|c|c|c|c|c|c|}
\hline Category of Disease & & Composition & & Organ & $\begin{array}{c}\text { Concoction } \\
\text { Technique }\end{array}$ & Usage \\
\hline Type of Treatment & Local Name & Scientific Name & Family & & & \\
\hline $\begin{array}{l}\text { Helps to treat consti- } \\
\text { pation }\end{array}$ & Barudata & $\begin{array}{l}\text { Euphorbia hetero- } \\
\text { phylla L. }\end{array}$ & Euphorbiaceae & Leaf & $\begin{array}{l}\text { Boiling tech- } \\
\text { nique }\end{array}$ & Drink \\
\hline Curing dysentery & Kalibau & $\begin{array}{l}\text { Hibischus tilliaceus } \\
\text { L. }\end{array}$ & Malvacae & Flower & $\begin{array}{l}\text { Boiling tech- } \\
\text { nique }\end{array}$ & Drink \\
\hline Curing diarrhea & Jono & $\begin{array}{l}\text { Imperata cylindrica } \\
\mathrm{L} .\end{array}$ & Poaceae & Root & $\begin{array}{l}\text { Boiling } \\
\text { technique and } \\
\text { then added by } \\
\text { brown sugar }\end{array}$ & Drink \\
\hline $\begin{array}{l}\text { Curing stomachache } \\
\text { and internal disease }\end{array}$ & Balaroa & $\begin{array}{l}\text { Kleinhovia hospita } \\
\text { L. }\end{array}$ & Sterculiaceae & Leaf & $\begin{array}{l}\text { Boiling tech- } \\
\text { nique }\end{array}$ & Drink \\
\hline $\begin{array}{l}\text { Curing diarrhea and } \\
\text { antimicrobial agent }\end{array}$ & Katumbara & Lantana camara L. & Verbenaceae & $\begin{array}{l}\text { Leaf } \\
\text { and } \\
\text { root }\end{array}$ & $\begin{array}{l}\text { Boiling tech- } \\
\text { nique }\end{array}$ & Drink \\
\hline $\begin{array}{l}\text { Help to accelerate } \\
\text { digestive system }\end{array}$ & Pala & $\begin{array}{l}\text { Myristica fragrans } \\
\text { Houtt. }\end{array}$ & Myristicaceae & Seed & $\begin{array}{l}\text { Boiling tech- } \\
\text { nique }\end{array}$ & Drink \\
\hline Curing diarrhea & Jambu Seed & $\begin{array}{l}\text { Psidium guajava } \\
\text { Linn. }\end{array}$ & Myrtacaea & Leaf & $\begin{array}{l}\text { Boiling tech- } \\
\text { nique }\end{array}$ & Drink \\
\hline $\begin{array}{l}\text { Curing diarrhea, } \\
\text { urolithiasis, fever, } \\
\text { and cough }\end{array}$ & Panuntu & $\begin{array}{l}\text { Phyllanthus niruri } \\
\text { L. }\end{array}$ & Phyllantaceae & $\begin{array}{l}\text { All } \\
\text { parts }\end{array}$ & $\begin{array}{l}\text { Boiling tech- } \\
\text { nique }\end{array}$ & Drink \\
\hline $\begin{array}{l}\text { Curing diarrhea and } \\
\text { body warmers }\end{array}$ & Kayu lana & $\begin{array}{l}\text { Tabernaemontana } \\
\text { pandacaqui Poir. }\end{array}$ & Apocynaceae & Root & $\begin{array}{l}\text { Boiling tech- } \\
\text { nique }\end{array}$ & Drink \\
\hline Stamina booster & Enau & $\begin{array}{l}\text { Arenga pinata } \\
\text { (Wurmb).Merr. }\end{array}$ & Arecaceae & $\begin{array}{l}\text { Plant } \\
\text { fluids }\end{array}$ & $\begin{array}{l}\text { Direct con- } \\
\text { sumption }\end{array}$ & Drink \\
\hline $\begin{array}{l}\text { Stamina booster and } \\
\text { detox agent }\end{array}$ & Kaluku & Cocos nucifera L. & Arecaceae & $\begin{array}{l}\text { Plant } \\
\text { fluids } \\
\text { and } \\
\text { bark }\end{array}$ & $\begin{array}{l}\text { Direct con- } \\
\text { sumption of } \\
\text { plant fluids } \\
\text { and bark is } \\
\text { boiled }\end{array}$ & Drink \\
\hline $\begin{array}{l}\text { Stamina booster and } \\
\text { detox agent }\end{array}$ & Sikuri & $\begin{array}{l}\text { Kaempferia galanga } \\
\text { L. }\end{array}$ & Zingiberaceae & $\begin{array}{l}\text { Rhi- } \\
\text { zome }\end{array}$ & $\begin{array}{l}\text { Boiling tech- } \\
\text { nique }\end{array}$ & Drink \\
\hline $\begin{array}{l}\text { Energy source, } \\
\text { staple food, cosmet- } \\
\text { ics and traditional } \\
\text { ritual material }\end{array}$ & $\mathrm{Pae}$ & Oryza sativa L. & Poaceae & Seed & $\begin{array}{l}\text { Grinding and } \\
\text { boiling tech- } \\
\text { nique }\end{array}$ & Eaten \\
\hline Stamina booster & Usuli & $\begin{array}{l}\text { Talinum panicula- } \\
\text { tum Gaertn. }\end{array}$ & Portulaceaea & Root & $\begin{array}{l}\text { Boiling tech- } \\
\text { nique }\end{array}$ & Drink \\
\hline $\begin{array}{l}\text { Stamina booster and } \\
\text { detox agent }\end{array}$ & Kula & $\begin{array}{l}\text { Zingiber officinale } \\
\text { Roxb.b. }\end{array}$ & Zingiberaceae & $\begin{array}{l}\text { Rhi- } \\
\text { zome }\end{array}$ & $\begin{array}{l}\text { Boiling tech- } \\
\text { nique, then } \\
\text { added sugar, } \\
\text { honey and egg }\end{array}$ & Drink \\
\hline $\begin{array}{l}\text { Appetite enhancer } \\
\text { for children }\end{array}$ & Balantua & $\begin{array}{l}\text { Alpinia galanga L. } \\
\text { Swartz. }\end{array}$ & Zingiberaceae & $\begin{array}{l}\text { Rhi- } \\
\text { zome }\end{array}$ & $\begin{array}{l}\text { Boiling tech- } \\
\text { nique }\end{array}$ & Drink \\
\hline $\begin{array}{l}\text { Immune system } \\
\text { booster }\end{array}$ & Banggudu & $\begin{array}{l}\text { Morinda citrifolia } \\
\text { L. }\end{array}$ & Rubiaceae & $\begin{array}{l}\text { Leaf } \\
\text { and } \\
\text { fruit }\end{array}$ & $\begin{array}{l}\text { Grating and } \\
\text { squeezing } \\
\text { technique }\end{array}$ & Drink \\
\hline Diabetes prevention & Pinahong & $\begin{array}{l}\text { Anredera cordifolia } \\
\text { (Ten.) Steenis. }\end{array}$ & Basellaceae & Leaf & $\begin{array}{l}\text { Boiling } \\
\text { technique and } \\
\text { then added by } \\
\text { honey }\end{array}$ & Drink \\
\hline
\end{tabular}


Slamet Ifandi, et al. / Biosaintifika 8 (1) (2016) 1-11

\begin{tabular}{|c|c|c|c|c|c|c|}
\hline Category of Disease & & Composition & & Organ & $\begin{array}{l}\text { Concoction } \\
\text { Technique }\end{array}$ & Usage \\
\hline Type of Treatment & Local Name & Scientific Name & Family & & & \\
\hline Diabetes prevention & Mimba & $\begin{array}{l}\text { Azadirachta indica } \\
\text { (Blume) Miq. }\end{array}$ & Meliaceae & Leaf & $\begin{array}{l}\text { Boiling tech- } \\
\text { nique }\end{array}$ & Drink \\
\hline $\begin{array}{l}\text { Diabetes wound } \\
\text { healing }\end{array}$ & Kunilola & $\begin{array}{l}\text { Curcuma domestica } \\
\text { Val. }\end{array}$ & Zingiberaceae & $\begin{array}{l}\text { Rhi- } \\
\text { zome }\end{array}$ & $\begin{array}{l}\text { Roasting and } \\
\text { grinding tech- } \\
\text { nique, added } \\
\text { by onion and } \\
\text { sugar }\end{array}$ & Affixed \\
\hline $\begin{array}{l}\text { To reduce blood } \\
\text { sugar level }\end{array}$ & Kayu momi & $\begin{array}{l}\text { Cinnamomum } \\
\text { zeylanicum } \mathrm{Bl} .\end{array}$ & Lauraceae & Bark & $\begin{array}{l}\text { Boiling tech- } \\
\text { nique }\end{array}$ & Drink \\
\hline $\begin{array}{l}\text { To reduce blood } \\
\text { sugar level }\end{array}$ & Tabaro & $\begin{array}{l}\text { Metroxylon sagu } \\
\text { Rottb. }\end{array}$ & Arecaceae & Sago & $\begin{array}{l}\text { Mashing and } \\
\text { Boiling tech- } \\
\text { nique }\end{array}$ & Eaten \\
\hline $\begin{array}{l}\text { To reduce blood } \\
\text { sugar level }\end{array}$ & Dale & Zea mays $\mathrm{L}$. & Poaceae & Seed & $\begin{array}{l}\text { Together with } \\
\text { rice, the seed } \\
\text { is washed and } \\
\text { then boiled }\end{array}$ & Eaten \\
\hline To cure skin ulcer & Kaki Kuda & $\begin{array}{l}\text { Centella asiatica }(\mathrm{L}) \\
\text { Urb. }\end{array}$ & Apiaceae & Leaf & $\begin{array}{l}\text { Grinding tech- } \\
\text { nique }\end{array}$ & Affixed \\
\hline To cure skin ulcer & Lanjima & $\begin{array}{l}\text { Purtulaca oleraceae } \\
\text { L. }\end{array}$ & Portulaceaea & Leaf & $\begin{array}{l}\text { Grinding tech- } \\
\text { nique }\end{array}$ & Affixed \\
\hline To cure skin ulcer & Valampanga & Physalis minima $\mathrm{L}$. & Solanacaeae & Leaf & $\begin{array}{l}\text { Grinding tech- } \\
\text { nique }\end{array}$ & Affixed \\
\hline To cure skin ulcer & $\begin{array}{l}\text { Sambi- } \\
\text { ralangi }\end{array}$ & $\begin{array}{l}\text { Zebrina pendula } \\
\text { Schnizl. }\end{array}$ & $\begin{array}{l}\text { Com- } \\
\text { melinaceaae }\end{array}$ & Leaf & $\begin{array}{l}\text { Leaf }+ \\
\text { curcumin }+ \\
\text { sugar, grinding } \\
\text { and boiling } \\
\text { technique }\end{array}$ & Affixed \\
\hline Urine flow enhancer & Salembangu & Lamium sp. & Lamiaceae & Leaf & $\begin{array}{l}\text { Boiling tech- } \\
\text { nique }\end{array}$ & Drink \\
\hline $\begin{array}{l}\text { Urine flow enhanc- } \\
\text { er, treat menstrual } \\
\text { problems, circulato- } \\
\text { ry system enhancer }\end{array}$ & Silevegie & $\begin{array}{l}\text { Fatoua pilosa } \\
\text { Gaud. }\end{array}$ & Moraceae & Root & $\begin{array}{l}\text { Root }+ \text { sugar } \\
+ \text { ash, Boiling } \\
\text { technique }\end{array}$ & Drink \\
\hline $\begin{array}{l}\text { Urinal system func- } \\
\text { tions maintenance }\end{array}$ & $\begin{array}{l}\text { Kumis kuc- } \\
\text { ing }\end{array}$ & $\begin{array}{l}\text { Orthosiphon arista- } \\
\text { tusMiq. }\end{array}$ & Labiataceae & $\begin{array}{l}\text { All } \\
\text { parts }\end{array}$ & $\begin{array}{l}\text { All parts }+5 \\
\text { other species, } \\
\text { Boiling tech- } \\
\text { nique }\end{array}$ & Drink \\
\hline Urine flow enhancer & Pepedi & Ruellia tuberosa L. & Acanthaceae & Leaf & $\begin{array}{l}\text { Boiling tech- } \\
\text { nique }\end{array}$ & Drink \\
\hline Burns remedial & Pia lei & Allium cepa L. & $\begin{array}{l}\text { Amaryllida- } \\
\text { ceae }\end{array}$ & Tuber & $\begin{array}{l}\text { Tuber + sugar, } \\
\text { mashing tech- } \\
\text { nique }\end{array}$ & Affixed \\
\hline To cure bruise & Kolontigi & $\begin{array}{l}\text { Aglalia odorata } \\
\text { Lour. }\end{array}$ & Meliaceae & Leaf & $\begin{array}{l}\text { Mashing tech- } \\
\text { nique }\end{array}$ & Affixed \\
\hline $\begin{array}{l}\text { To cure bruise in } \\
\text { breast }\end{array}$ & Rarayo & $\begin{array}{l}\text { Cladodendron ser- } \\
\text { ratum } \mathrm{L} \text {. }\end{array}$ & Verbenaceae & Leaf & $\begin{array}{l}\text { Grinding tech- } \\
\text { nique }\end{array}$ & Affixed \\
\hline Burns remedial & Kadambuku & $\begin{array}{l}\text { Justicia gendarussa } \\
\text { Burm.f }\end{array}$ & Achantaceae & Leaf & $\begin{array}{l}\text { Grinding tech- } \\
\text { nique }\end{array}$ & Smeared \\
\hline $\begin{array}{l}\text { To cure cough up } \\
\text { phlegm in children } \\
\text { (expectorant) }\end{array}$ & Katimunda & Abrus precatorius $\mathrm{L}$. & Fabaceae & Leaf & $\begin{array}{l}\text { Leaf + cumin, } \\
\text { mashing and } \\
\text { squeezing } \\
\text { technique }\end{array}$ & Drink \\
\hline
\end{tabular}


Slamet Ifandi, et al. / Biosaintifika 8 (1) (2016) 1-11

\begin{tabular}{|c|c|c|c|c|c|c|}
\hline Category of Disease & & Composition & & Organ & $\begin{array}{c}\text { Concoction } \\
\text { Technique }\end{array}$ & Usage \\
\hline Type of Treatment & Local Name & Scientific Name & Family & & & \\
\hline $\begin{array}{l}\text { Bad breath remover } \\
\text { and curing the } \\
\text { cough }\end{array}$ & Pia mputi & $\begin{array}{l}\text { Allium ascolonicum } \\
\text { L. }\end{array}$ & $\begin{array}{l}\text { Amaryllida- } \\
\text { ceae }\end{array}$ & Tuber & $\begin{array}{l}\text { Tubermashing } \\
\text { and squeezing } \\
\text { technique }\end{array}$ & Drink \\
\hline Expectorant & Rayambone & $\begin{array}{l}\text { Amaranthus spino- } \\
\text { sus L. }\end{array}$ & $\begin{array}{l}\text { Amarantha- } \\
\text { ceae }\end{array}$ & Leaf & $\begin{array}{l}\text { Boiling tech- } \\
\text { nique, added } \\
\text { by lime water }\end{array}$ & Drink \\
\hline $\begin{array}{l}\text { To cure tonsil irrita- } \\
\text { tion and cough up } \\
\text { phlegm }\end{array}$ & Lemo nipis & $\begin{array}{l}\text { Citrus aurantifolia } \\
\text { (Christm.)Swingle }\end{array}$ & Rutaceae & Fruit & $\begin{array}{l}\text { Squeezing } \\
\text { technique add- } \\
\text { ed by ketchup } \\
\text { and salt }\end{array}$ & Drink \\
\hline $\begin{array}{l}\text { To cure cough and } \\
\text { sore throat }\end{array}$ & Paria & $\begin{array}{l}\text { Momordica charan- } \\
\text { tia L. }\end{array}$ & Cucurbitaceae & Leaf & $\begin{array}{l}\text { Boiling tech- } \\
\text { nique }\end{array}$ & Drink \\
\hline To cure cough & Timbaya & $\begin{array}{l}\text { Solanum rudepan- } \\
\text { num Dunal. }\end{array}$ & Solanacaeae & Leaf & $\begin{array}{l}\text { Boiling tech- } \\
\text { nique }\end{array}$ & Drink \\
\hline To cure headache & Sarikaya & $\begin{array}{l}\text { Annona squamosa } \\
\text { L. }\end{array}$ & Annonaceae & Leaf & $\begin{array}{l}\text { Boiling tech- } \\
\text { nique }\end{array}$ & Drink \\
\hline To cure headache & Kena & $\begin{array}{l}\text { Andrographis pa- } \\
\text { niculata Burn.f }\end{array}$ & Achantaceae & Leaf & $\begin{array}{l}\text { Boiling tech- } \\
\text { nique }\end{array}$ & Drink \\
\hline To cure headache & Tambajara & Erigeron sp. & Asteraceae & Leaf & $\begin{array}{l}\text { Boiling tech- } \\
\text { nique }\end{array}$ & Drink \\
\hline $\begin{array}{l}\text { Fever healer and to } \\
\text { cure internal disease }\end{array}$ & Lengaru & $\begin{array}{l}\text { Alstonia scholaris } \\
\mathrm{R} . \mathrm{Br}\end{array}$ & Apocynaceae & Bark & $\begin{array}{l}\text { Boiling tech- } \\
\text { nique }\end{array}$ & Drink \\
\hline Fever healer & Kayu lei & Bridelia sp. & Phyllanthaceae & Leaf & $\begin{array}{l}\text { Boiling tech- } \\
\text { nique }\end{array}$ & Drink \\
\hline Fever healer & Maku & $\begin{array}{l}\text { Syzygium malac- } \\
\text { cense (L.) Merr \& } \\
\text { Perry }\end{array}$ & Myrtacaea & Bark & $\begin{array}{l}\text { Boiling tech- } \\
\text { nique }\end{array}$ & Drink \\
\hline $\begin{array}{l}\text { To cure skin ulcer } \\
\text { and skin disease }\end{array}$ & Kamonji & $\begin{array}{l}\text { Artocarpus commu- } \\
\text { nis J.R \& G.Frost }\end{array}$ & Moraceae & Leaf & $\begin{array}{l}\text { Immersion } \\
\text { technique }\end{array}$ & Rubbed \\
\hline $\begin{array}{l}\text { To cure skin ulcer } \\
\text { and skin disease }\end{array}$ & Roviga & $\begin{array}{l}\text { Calotropis gigantea } \\
\text { L.W.T.Aiton }\end{array}$ & Asclepiadaceae & Leaf & $\begin{array}{l}\text { Mashing tech- } \\
\text { nique added by } \\
\text { coconut milk }\end{array}$ & Rubbed \\
\hline Anti-toxin & Gampaya & Carica papaya $\mathrm{L}$. & Caricaceae & Leaf & $\begin{array}{l}\text { Mashing and } \\
\text { squeezing tech- } \\
\text { nique }\end{array}$ & Drink \\
\hline $\begin{array}{l}\text { Anti-toxin, condi- } \\
\text { ment, and tradition- } \\
\text { al ritual material }\end{array}$ & Tumbavani & $\begin{array}{l}\text { Cymbopogon citra- } \\
\text { tus (DC.) Stapf. }\end{array}$ & Poaceae & Stem & $\begin{array}{l}\text { Boiling tech- } \\
\text { nique }\end{array}$ & Drink \\
\hline $\begin{array}{l}\text { Helps body as anti- } \\
\text { toxinagent }\end{array}$ & Loka & $\begin{array}{l}\text { Musa paradisiaca } \\
\text { L. }\end{array}$ & Musaceae & Stem & $\begin{array}{l}\text { Boiling tech- } \\
\text { nique }\end{array}$ & Drink \\
\hline $\begin{array}{l}\text { To reduce high } \\
\text { blood pressure }\end{array}$ & Alpukat & $\begin{array}{l}\text { Persea americana } \\
\text { Mill. }\end{array}$ & Lauraceae & Leaf & $\begin{array}{l}\text { Boiling tech- } \\
\text { nique }\end{array}$ & Drink \\
\hline $\begin{array}{l}\text { To reduce high } \\
\text { blood pressure }\end{array}$ & Labu sia & $\begin{array}{l}\text { Sechium edule } \\
\text { (Jacq).Swarrt. }\end{array}$ & Cucurbitaceae & Fruit & $\begin{array}{l}\text { Grating and } \\
\text { squeezing tech- } \\
\text { nique }\end{array}$ & Drink \\
\hline $\begin{array}{l}\text { Facilitating breast- } \\
\text { feeding and postpar- } \\
\text { tum treatment }\end{array}$ & Gedi & $\begin{array}{l}\text { Abelmoschus mani- } \\
\text { hot L. }\end{array}$ & Malvaceae & Leaf & $\begin{array}{l}\text { Boiling tech- } \\
\text { nique }\end{array}$ & Drink \\
\hline $\begin{array}{l}\text { Postpartum treat- } \\
\text { ment }\end{array}$ & Asam jawa & $\begin{array}{l}\text { Tamarindus indica } \\
\text { L. }\end{array}$ & $\begin{array}{l}\text { Caesalpini- } \\
\text { aceae }\end{array}$ & Fruit & $\begin{array}{l}\text { Immersion } \\
\text { technique } \\
\text { added by } \\
\text { honey and egg }\end{array}$ & Drink \\
\hline
\end{tabular}


Slamet Ifandi, et al. / Biosaintifika 8 (1) (2016) 1-11

\begin{tabular}{|c|c|c|c|c|c|c|}
\hline Category of Disease & & Composition & & Organ & $\begin{array}{l}\text { Concoction } \\
\text { Technique }\end{array}$ & Usage \\
\hline Type of Treatment & Local Name & Scientific Name & Family & & & \\
\hline To cure asthma & Taipa & Mangifera indica $\mathrm{L}$ & Anacardiaceae & Bark & $\begin{array}{l}\text { Boiling tech- } \\
\text { nique, added } \\
\text { by ginger }\end{array}$ & Drink \\
\hline To cure asthma & Loka Ibo & Musa sp. & Musaceae & Fruit & $\begin{array}{l}\text { Mashing and } \\
\text { squeezing tech- } \\
\text { nique }\end{array}$ & Drink \\
\hline To cure asthma & Sirih hutan & $\begin{array}{l}\text { Piper caducibracte- } \\
\text { um C.DC }\end{array}$ & Piperaceae & Leaf & $\begin{array}{l}\text { Boiling tech- } \\
\text { nique }\end{array}$ & Drink \\
\hline $\begin{array}{l}\text { To reduce gastric } \\
\text { acid }\end{array}$ & Karyango & Acorus calamus L. & Araceae & $\begin{array}{l}\text { Rhi- } \\
\text { zome }\end{array}$ & $\begin{array}{l}\text { Grinding and } \\
\text { squeezing tech- } \\
\text { nique }\end{array}$ & Drink \\
\hline $\begin{array}{l}\text { To cure stomach } \\
\text { ulcer }\end{array}$ & Tabanjaia & $\begin{array}{l}\text { Erigeron sumatran- } \\
\text { sis L. }\end{array}$ & Asteraceae & Bark & $\begin{array}{l}\text { Boiling tech- } \\
\text { nique }\end{array}$ & Drink \\
\hline $\begin{array}{l}\text { To cure stomach } \\
\text { ulcer }\end{array}$ & Kayu Jawa & $\begin{array}{l}\text { Lannea coromandel- } \\
\text { ica (Houtt.) Merr. }\end{array}$ & Anacardiaceae & Bark & $\begin{array}{l}\text { Boiling tech- } \\
\text { nique, added } \\
\text { sugar }\end{array}$ & Drink \\
\hline To cure toothache & Tavumboa & $\begin{array}{l}\text { Crinum asiaticum } \\
\text { L. }\end{array}$ & $\begin{array}{l}\text { Amaryllida- } \\
\text { ceae }\end{array}$ & Root & $\begin{array}{l}\text { Mashing tech- } \\
\text { nique }\end{array}$ & $\begin{array}{l}\text { Affixed } \\
\text { onto ache } \\
\text { tooth }\end{array}$ \\
\hline $\begin{array}{l}\text { To cure a toothache } \\
\text { and traditional ritual } \\
\text { material }\end{array}$ & Kulalo & Jatropha curcas L. & Euphorbiaceae & Sap & $\begin{array}{l}\text { Direct con- } \\
\text { sumption }\end{array}$ & $\begin{array}{l}\text { Dropped } \\
\text { onto ache } \\
\text { tooth }\end{array}$ \\
\hline Joint pain treatment & Rica jawa & Piper nigrum L. & Piperaceae & Seed & $\begin{array}{l}\text { Mashing tech- } \\
\text { nique added } \\
\text { by egg }\end{array}$ & Drink \\
\hline $\begin{array}{l}\text { Rheumatic pain } \\
\text { treatment }\end{array}$ & Sibalaya & Sida acuta Burm.f. & Malvaceae & Leaf & $\begin{array}{l}\text { Boiling tech- } \\
\text { nique }\end{array}$ & Drink \\
\hline To cure earache & Tara & $\begin{array}{l}\text { Ananas camosus L. } \\
\text { Merr. }\end{array}$ & Bromeliaceae & Fruit & $\begin{array}{l}\text { Grating and } \\
\text { squeezing } \\
\text { technique }\end{array}$ & $\begin{array}{l}\text { Dropped } \\
\text { into ache } \\
\text { ear }\end{array}$ \\
\hline To cure jaundice & Valangguni & $\begin{array}{l}\text { Arcangelisia flava } \\
\text { L. Merr. }\end{array}$ & $\begin{array}{l}\text { Menisperma- } \\
\text { ceae }\end{array}$ & Root & $\begin{array}{l}\text { Boiling tech- } \\
\text { nique }\end{array}$ & Drink \\
\hline Throat soothing & Tamadia & $\begin{array}{l}\text { Artemisia vulgaris } \\
\text { L. }\end{array}$ & Asteraceae & Leaf & $\begin{array}{l}\text { Mashing and } \\
\text { squeezing } \\
\text { technique }\end{array}$ & Drink \\
\hline $\begin{array}{l}\text { To cure internal } \\
\text { disease }\end{array}$ & Baliura & Begonia sp. & Bignoniaceae & Leaf & $\begin{array}{l}\text { Boiling tech- } \\
\text { nique }\end{array}$ & Drink \\
\hline To cure impotence & Patoko & Eleusine indica L. & Poaceae & Leaf & $\begin{array}{l}\text { Boiling tech- } \\
\text { nique }\end{array}$ & Drink \\
\hline Fever healer & Siranindi & $\begin{array}{l}\text { Kalanchoe pinnata } \\
\text { Pers. }\end{array}$ & Crasulaceae & Leaf & $\begin{array}{l}\text { Mashing and } \\
\text { squeezing } \\
\text { technique }\end{array}$ & Drink \\
\hline To cure scabies & Paria hutan & $\begin{array}{l}\text { Momordica bal- } \\
\text { samina L. }\end{array}$ & Cucurbitaceae & Leaf & $\begin{array}{l}\text { Mashing tech- } \\
\text { nique, added } \\
\text { by brown sugar }\end{array}$ & Smeared \\
\hline To cure sore eyes & Kelo & $\begin{array}{l}\text { Moringa oleifera } \\
\text { Limk. }\end{array}$ & Moringacaea & Leaf & $\begin{array}{l}\text { Immersion } \\
\text { technique }\end{array}$ & $\begin{array}{l}\text { Dropped } \\
\text { onto eyes }\end{array}$ \\
\hline To cure TBC & Botedala & Tournefortiasp. & Boraginaceae & Leaf & $\begin{array}{l}\text { Boiling tech- } \\
\text { nique }\end{array}$ & Drink \\
\hline $\begin{array}{l}\text { To cure vaginal } \\
\text { discharge }\end{array}$ & Sirih & Piper betle $\mathrm{L}$. & Piperaceae & Leaf & $\begin{array}{l}\text { Boiling tech- } \\
\text { nique }\end{array}$ & Drink \\
\hline
\end{tabular}


Slamet Ifandi, et al. / Biosaintifika 8 (1) (2016) 1-11

\begin{tabular}{|c|c|c|c|c|c|c|}
\hline \multirow{2}{*}{$\begin{array}{l}\text { Category of Disease } \\
\text { Type of Treatment }\end{array}$} & \multirow[b]{2}{*}{ Local Name } & \multicolumn{2}{|l|}{ Composition } & \multirow[t]{2}{*}{ Organ } & \multirow[t]{2}{*}{$\begin{array}{l}\text { Concoction } \\
\text { Technique }\end{array}$} & \multirow[t]{2}{*}{ Usage } \\
\hline & & Scientific Name & Family & & & \\
\hline $\begin{array}{l}\text { To kill Tinea vesicolor } \\
\text { (skin fungus) }\end{array}$ & $\begin{array}{l}\text { Kayu ma- } \\
\text { nuru }\end{array}$ & Senna alata L. & Fabaceae & Leaf & $\begin{array}{l}\text { Mashing tech- } \\
\text { nique }\end{array}$ & Rubbed \\
\hline $\begin{array}{l}\text { To reduce high level } \\
\text { of uric acid }\end{array}$ & Silaguri & Sida rhombifolia L. & Malvaceae & $\begin{array}{l}\text { All } \\
\text { parts }\end{array}$ & $\begin{array}{l}\text { Immersion } \\
\text { technique }\end{array}$ & Drink \\
\hline Sore throat drugs & Calicope & $\begin{array}{l}\text { Syzygium cumini L. } \\
\text { Skeels. }\end{array}$ & Myrtacaea & Bark & $\begin{array}{l}\text { Boiling tech- } \\
\text { nique }\end{array}$ & Drink \\
\hline Anti-cancer & Pesumu & $\begin{array}{l}\text { Xanthosoma viola- } \\
\text { ceum L. }\end{array}$ & Asteraceae & Leaf & $\begin{array}{l}\text { Boiling tech- } \\
\text { nique }\end{array}$ & Drink \\
\hline Sedative drugs & Kalagi & $\begin{array}{l}\text { Zingiber montanum } \\
\text { (Link). Roxb }\end{array}$ & Zingiberaceae & $\begin{array}{l}\text { Rhi- } \\
\text { zome }\end{array}$ & $\begin{array}{l}\text { Mashing and } \\
\text { squeezing } \\
\text { technique }\end{array}$ & Drink \\
\hline
\end{tabular}

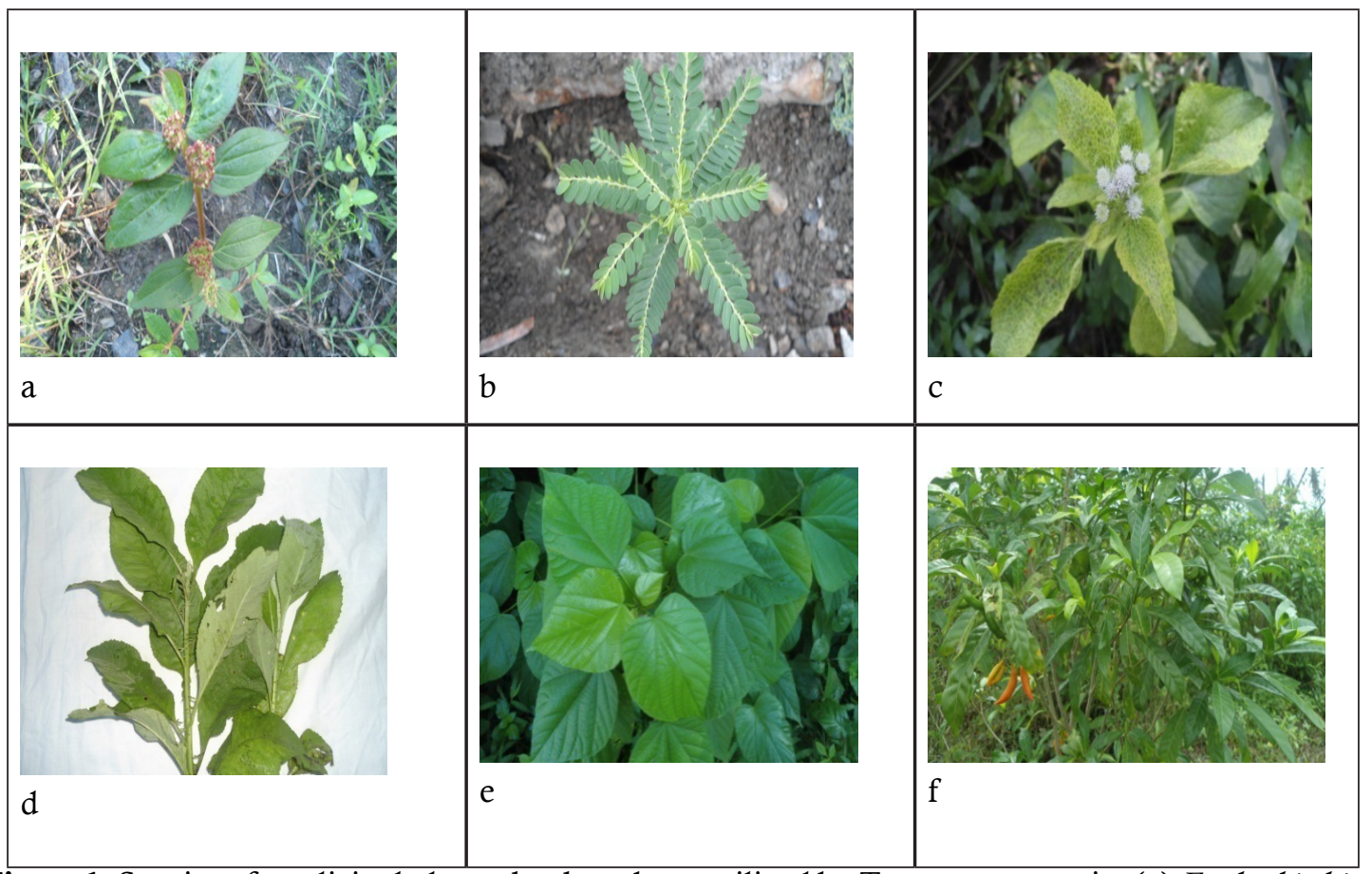

Figure 1. Species of medicinal plants that have been utilized by Tompu community (a).Euphorbia hirta L. (b). Phyllanthus niruri L. (c). Ageratum conyzoides L. (d). Blumea balsaminifera (L.) DC. (e). Kleinhovia hospita L. (f). Tabernaemontana pandacaqui Poir.

Based on the interview results, it was known that there were several species with a high value of medicinal benefits in Tompu. Such as Euphorbia hirta L. has been used as anti-venom, fever, and asthma. Phyllanthus niruri L. has been employed to treat diarrhea, bladder stones, fever, and cough. Ageratum conyzoidesL. as an antipoison of insect bites and material for traditional rituals. Blumea balsaminifera L. (DC). Has been applied for curing the diarrhea and another internal disease. Kleinhovia hospita L. also has been utilized to treat abdominal pain and internal disease medicine. Tabernaemontana panda Aqui has been applied to cope with diarrhea and body warmers
(Figure 1).

The use of Mantalalu (Euphorbia hirta L.) leaves has been aimed to overcome the symptoms of bladder stone disease, appendicitis, abdominal pain, diarrhea, anti-toxins, fever and asthma. The leaves are being cleaned and then it is coupled with 1 liter of water and then boiled. The boiled water is added to honey and chicken egg. In fact, according to sando, all parts of the plant can be used as a medicine and any plant species must be combined in pairs. The goal is that the properties will be more effective in curing the disease. This plant also has been widely used as a traditional medicine in countries which located in tropical 
regions, such as Africa, Asia, South America, and Australia. In Chinese society, all parts of this plant are used to treat digestive disorders such as diarrhea, ulcers, heartburn and vomiting (Huang et al., 2012). Gayathri \& Ramesh (2013) research results showed that the leaf extracts have antibacterial properties against pathogenic microorganisms.

Phyllanthus niruri L. is commonly used to treat diarrhea, as an antimicrobial agent, to cure bladder stones, cough, fever and diarrhea. The plant leaves are often used. The utilization of this plant consists of two, outer usage and inner usage. Leaves are grinded until finely ground and then compressed on the head, whereas the inner usage is by consuming it. Before consuming it, it combined with other plants and then boiled. Boiled water sometimes also used to treat ulcers. In Indonesia, $P$ niruri $\mathrm{L}$. is one of the herbs that have been used for generations for the treatment of various diseases such as diuretics, antioxidant, antimicrobial, anti-inflammatory, anti-diabetic, antipyretic, appetite enhancer, heat in the stomach and tonic (Alegantina et al, 2014). According to Baskaran et al. (2010). P. niruri has several pharmacological properties that are used to treat lesions, urolithiasis, urinary tract infections and stomach diuretic. This plant also has been used since ancient times for treatment of jaundice and liver disorders.

Ageratum conyzoidesL. or salembangu is one species that has important benefits for Tompu society. Leaves of this plant are used to cure cough, abdominal pain, internal medicine, sore throat, anti-toxin and wound healer. The leaves are boiled and then it is added to sugar and honey, then it directly consumed. Besides that, it is often used as ritual ceremonies of rice farming. At the open- ing of the rice fields, salembangu is always placed in some corners of the farm. Based on the interview results with sando, it can remove pests for plant resignations. In India, people use this plant as anti-bacterial agent, anti-dysentery, and antilytic. While in Asia, South America and Africa, they use leaves of $A$. conyzoides to treat pneumonia, wound healing, treating fever, rheumatism, headaches and digestive disorders (Amadi, et al., 2012). Shekhar \& Anju (2012) showed that almost all the parts of $A$. conyzoides components are alkaloids, flavonoids, tannins, saponins, glycosides, resin, and phenol functions as pharmacological activity and insecticide

Blumea balsamifera (L.) DC or Sivumboa is often used as a traditional medicine to cure diarrhea and other internal diseases. Usually, young leaves are chosen as drugs. Boiling method is applied to obtain decoction water. Decoction is let to stand for 15 minutes and then it consumed directly. Today, most people in Tompu have planted this species around their settlements. The leaves of B. balsamifera (L.) DC.were used by Sundanese people as an ulcer drug. Javanese people utilized it to overcome fever and malaria, and the community in Southeast Sulawesi, in Wawoni Island are using leaves of this plant for the treatment of postpartum and body warmers (Rahayu. et al. 2006). Local communities in Thailand and China use this plant for the treatment of wounds and kidney disorders. It also reported as an antifungal, antibacterial, antifebrile, coryza, fever, influenza and coughing (Lee, et al, 2012).

Utilization of balaroa (Kleinhovia hospita L.) as a traditional medicine by Tompu society, it used to treat stomachache and internal disease. The boiling technique is used for treating the leaves before consumption. It can be mixed with ot-

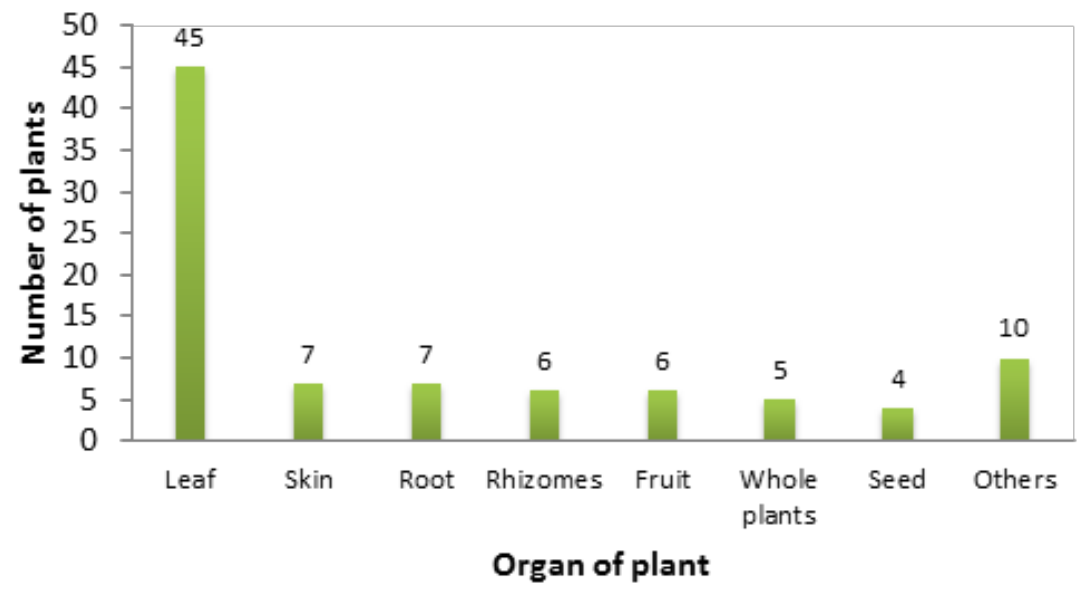

Figure 2. Amount of organ and part of plant for medicinal purpose in Tompu society 


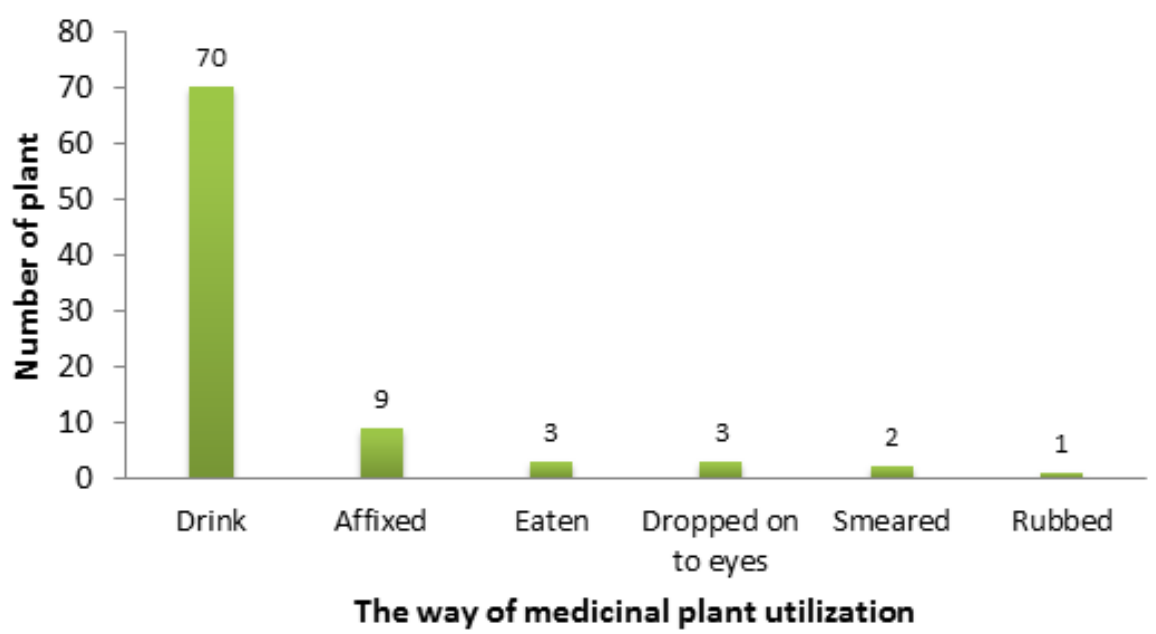

Figure 3. The way of medicinal plant utilization by Tompu community

her plants. Traditionally, this plant has been used as medicine by the general public, especially in the Southeast, Central and South Sulawesi. It is believed to have medicinal properties that can cure liver disease. Rahayu et al, (2006) stated that the leaves and bark of this plant contain cyanogenic compounds which are efficacious as a repellent of ectoparasites such as ticks. While the leaf extract has an anti-tumor activity.

Tabernaemontana pandacaqui Poir Poire plant is known as a major source of modern medicine. The root of this plant has been applied to treat diarrhea and as body warmers. In China and Thailand, this plant is a traditional medicine used for the treatment of fever, pain and dysentery (Pratchayasakul et.al., 2008). Based on the results of the study, it showed that leaf is the most widely utilized plant organ for drug formulation. It followed by bark and root and then flower and stem, respectively (Figure 2).

Part used include leaves, stems, tubers, roots, rhizomes, stems, bark, flowers, fruits, seeds, sap, the entire section. Overall plant organs which are often used by people Tompu as medicine are the leaves. According to sando leaves have a high level of effectiveness as drugs. Additionally leaf plant is part of the most numerous and easy to get. The leaves also have high regeneration for re-sprout and does not give a great influence on the growth of a plant even though the leaves is where photosynthesis (Anggana, 2011).

According to Tangiitman et al. (2015), the leaves are part of the plant used the most widely used as a traditional medicine in comparison with other parts. Many studies have been conducted in various regions also showed the dominance of leaf organs in the use of drugs. Leaves are the primary photosynthetic organs of plants and is considered a key component of the natural pharmacy for the synthesis of bioactive components such as pharmacologically active ingredients which can be used to cure certain diseases. Tompu community usually uses the medicinal plants by direct and indirect consumption with pre-treatment before it. Herbal drink is the most popular form of consumption way.

Based on the interview results with treatment master, the popular way of medicinal treatment in Tompu society are divided into two. They are direct and indirect uses. Direct use is generally in the form of herbal drink, whereas the indirect use is in the form of rubbing method. The direct way using herbal drink is considered to be more effective to accelerate the healing process, and it is easier and practical to be applied to specific categories of the disease. An herbal drink made from the leaves is also reported as one of the practical ways in serving and using of medicinal plants that have been understood and applied by various groups of traditional society (Maroyi, 2013).

\section{CONCLUSION}

The management and the use of medicinal plants by Tompu community represented their knowledge of medicinal plant utilization. The level of knowledge of Tompu society was measured according to their understanding and views on cultural customs and traditions that have been passed down from ancestors through generations to generations. Based on the obtained results, from 90 species of medicinal plants, 18 of them were used to treat gastrointestinal problems by Tompu people. Tompu society uses medicinal plants in a way of directly or indirectly applicati- 
on. People consume it directly by oral and use it indirectly by rubbing and affixing. The most used organ of the plant is the leaves. The benefits of this research to the development of science are expected to be complete scientific data regarding the utilization of medicinal plants natural resources on Tribal society Kaili in Tompu

\section{REFERENCES}

Alegantina, Sukmayati, Setyorini, H. A. \& Triwahyuni. (2014). Pengujian Mutu dan Penetapan Kadar Filantin Pada Ekstrak Etanol Herba Meniran (Phyllanthus niruri Linn.). Pusat Biomedis dan Teknologi Dasar Kesehatan, 43(1), 11-16.

Amadi, B. A., Duru, M. K. C. \& Agomuo, E. N. (2012). Chemical profilesof leaf, stem, root and flower of Ageratum conyzoides. Department of Biochemistry, Imo State University, Owerri, Imo State, Nigeria. Asian Journal of Plant Science and Research, 2(4), 428-432.

Anggana, A. F. (2011). Kajian Etnobotani Masyarakat di Sekitar TNGM (Studi kasus di Desa Umbulharjo, Sidorejo, Wonodoyo dan Ngablak). Skripsi. Bogor: Fakultas Kehutanan. IPB.

Baskaran, M. Periyasamy L. \& Rajagopalan. (2010). Effect of Phyllanthus niruri $\mathrm{n}$ alcohol and polyunsaturated fatty acid induced oxidative stress in liver. Department of Biochemistry and Molecular Biology, Pondicherry University, Kalapet, Tamilnadu, India. International Journal of Pharmacy and Pharmaceutical Sciences, 2(4), 5862.

Gayathri \& Ramesh, K. V. (2013). Antifungal activity of Euphorbia hirta L. inflorescence extract against Aspergillus flavus A mode of action study. Department of Plant Biology and Plant Biotechnology Tamil Nadu. International Journal of Current Microbiology and Applied Sciences, 2(4), 31-37.

Hariyadi, B. (2011). Obat Rajo Obat Ditawar : Tumbuhan Obat dan Pengobatan Tradisional Masyarakat Serampas Jambi. Biospecies, 4(2), 29 34.

Hartanto, S., Fitmawati \& Sofiyanti, N. (2014). Studi Etnobotani Famili Zingiberaceae dalam Kehidupan Masyarakat Lokal di Kecamatan Pangean Kabupaten Kuantan Singingi, Riau. Biosaintifika: Journal of Biology \& Biology Education, 6(2), 98-108.

Huang, L., Chen, S. \& Yang, M. (2012). Euphorbia hirta (Feiyangcao): A review on its ethnopharmacology, phytochemistry and pharmacology. Institute of Medicinal Plant Development, Chinese Academy of Medical Sciences and Peking Union Medical College, Beijing, China. Journal of Medicinal Plants Research. 6(39), 5176-5185.

Jumari, Setiadi \& Purwanto, Y. (2012). Etnobiologi
Masyarakat Samin. Disertasi. Program Pasca Sarjana IPB. Bogor.

Jhosi, Wijaya, Sirait \& Mulyoutami, E. (2004). Indegenous System and Ecologycal Knowledge Among Dayak People in Kutai Barat, East Kalimantan. Bogor World Agroforesti Centre-ICRAF Working Paper.

Lee, D. G., Mok, S. M., Choi, C., Cho, E. J., Kim, H. Y. \& Sanghyun, L. (2012). Analysis of apigenin in Blumea balsamifera Linn DC. and its inhibitory activity against aldose reductase in rat lens. Department of Integrative Plant Science, Chung-Ang University, Anseong, South Korea. Journal of Agricultural Chemistry and Environment, 1(1), 28-33.

Maroyi, A. (2013). Traditional use of medicinal plants in south-central Zimbabwe: review and perspectives. Journal of Ethnobiology and Ethnomedicine. 9(1), 9-31.

Martin, G. J. (2004). Ethnobotany. A methods manual. People and plants conservation series. WWF.

Nasab, F. K., \& Khosravi, A. R. (2014). Ethnobotanical study of medicinal plants of Sirjan in Kerman Province, Iran. Journal of ethnopharmacology, 154(1), 190-197.

Pratchayasakul, Pongchaidecha, Chattipakorn, N., \& Chattipakorn, S. (2008). Ethnobotany \& Ethnopharmacology of Tabernaemontana divaricata. Indian Journal of Medical Research. 127(4), 317-335.

Rahayu, M., Sunarti, S., Sulistiarini, D., \& Prawiroatmodjo, S. (2006). Pemanfaatan Tumbuhan Obat secara Tradisional oleh Masyarakat Lokal di Pulau Wawoni, Sulawesi Tenggara. Jurnal Biodiversitas, 7(3), 245-250.

Shanthi, R. V., Jumari, \& Izzati, M. (2014). Studi Etnobotani Pengobatan Tradisional untuk Perawatan Wanita di Masyarakat Keraton Surakarta Hadiningrat. Biosaintifika: Journal of Biology \& Biology Education, 6(2), 61-69.

Shekhar, T. C. \& Anju, G. (2012). A Comprehensive Review on Ageratum conyzoides Linn.(Goat weed) Division of Pharmaceutical Sciences, Shri Guru Ram Rai Institute of Technology and Science, Patel Nagar, Dehradun, Uttarakhand, India. International Journal of Pharmaceutical and Phytopharmacological Research. 1(6), 391-395.

Tangjitman, K., Wongsawad, C., Kamwong, K., Sukkho, T., \& Trisonthi, C. (2015). Ethnomedicinal plants used for digestive system disorders by the Karen of northern Thailand. Journal of ethnobiology and ethnomedicine, 11(1), 11-27.

Yuniati, E., \& Alwi, M. (2015). Etnobotani Keanekaragaman Jenis Tumbuhan Obat Tradisional dari Hutan di Desa Pakuli Kecamatan Gumbasa Kabupaten Donggala, Sulawesi Tengah. Biocelebes, 4(1), 69-75. 